\title{
Analysis of the Amplitude Changes and Baseline Drift of Respiratory Motion during Liver Stereotactic Body Radiation Therapy Based on Intra-Fraction CBCT
}

\section{Lu Zeng}

Sichuan University West China Hospital

\section{Xin Wang}

Sichuan University West China Hospital

Jidan Zhou Zhou

Sichuan University West China Hospital

Pan Gong

Sichuan University West China Hospital

\section{Xuetao wang}

Sichuan University West China Hospital

\section{Xiaohong Wu}

Panzhihua University

\section{Zhonghua Deng}

Sichuan University West China Hospital

\section{Bin Li}

Sichuan University West China Hospital

Renming Zhong ( $\sim$ zrm_100@163.com)

Sichuan University West China Hospital https://orcid.org/0000-0002-1429-6469

\section{Research}

Keywords: Intra-fraction CBCT, Liver SBRT, Amplitude changes and baseline drifts, PTV margin

Posted Date: July 7th, 2020

DOI: https://doi.org/10.21203/rs.3.rs-40351/v1

License: (c) (i) This work is licensed under a Creative Commons Attribution 4.0 International License. Read Full License 


\section{Abstract}

Purpose: To evaluate the amplitude changes and baseline drift of respiratory motion in liver stereotactic body radiation therapy based on intra-fraction cone beam CT (CBCT).

Materials and methods: Twenty-four liver SBRT patients underwent a four-dimensional computed tomography (4D CT) scan, inter-fraction cone-beam computed tomography (CBCT), and intra-fraction CBCT to evaluate the amplitude changes and baseline drift of respiratory motion. The amplitude changes were defined as the variations between the amplitude measured in the 4D CT and those measured with fluoroscopy in inter- and intra-fraction СВCT. The baseline drifts were defined as the difference between the liver position errors and the setup errors in inter- and intra-fraction CBCT. Manual registration of the liver contour was performed to obtain liver position errors, and bone registration was performed to obtain setup errors. Meanwhile, the correlation among the liver position errors, the relative diaphragm position, and the amplitude changes was evaluated.

Results: The systematic and random errors of the baseline drifts for intra-fraction CBCT in the mediallateral (ML), superior-inferior (SI), and anterior-posterior (AP) views were 0.99 and $1.6 \mathrm{~mm}, 2.03$ and 2.46 $\mathrm{mm}$, and 1.02 and $2.07 \mathrm{~mm}$, respectively. The corresponding PTV margin was $3.61 \mathrm{~mm}, 6.8 \mathrm{~mm}$, and 4.00 $\mathrm{mm}$, respectively. The amplitude variation ranged from $-169.33 \%$ to $65.47 \%$ in inter-fraction CBCT and from $-171.04 \%$ to $60.00 \%$ in intra-fraction CBCT. Inter-fraction liver position errors were significantly related to the setup errors. The relative diaphragm position was not statistically related to the baseline drifts in the inter-fraction nor in the intra-fraction.

Conclusions: Using intra-fraction $\mathrm{CBCT}$, significant amplitude variations and baseline drifts of respiratory motion were found compared with those in 4D CT. The relative diaphragm position in a single breathing cycle cannot reflect the liver position during dose delivery. At least a $6.8 \mathrm{~mm}$ PTV margin in the SI direction is recommended to compensate for the baseline drift.

\section{Background:}

Stereotactic body radiation therapy (SBRT) has become the main treatment method for inoperable liver cancer and liver metastases and can significantly improve the local control rate of tumour in patients. The dose gradient around the target area of SBRT technology changes sharply, and a small position deviation can cause a significant dose change in the target area. The target area of the liver is affected by inter-fraction errors (mainly the setup errors) and respiratory motion. Therefore, image guided radiotherapy (IGRT) and respiratory motion management strategies must be adopted to correct these errors and improve the accuracy of radiotherapy [1].The IGRT strategies include cone-beam CT (CBCT), KV plain film [2], MR [3] and EPID, etc. Respiratory motion management includes expanded margin under free breathing, 4D CT [4], tracking[5], gating, and deep inspiration breath-hold (DIBH) [6]. CBCT-guided $\mathrm{DIBH}$ technology can reduce the patient's breathing movement; however, some patients do not tolerate this well, and a larger PTV margin is needed to compensate for the variation between different breath- 
holds [4]. Gating and tracking technologies usually require additional auxiliary equipment and implantation benchmarks. Therefore, 4D CT is still the most popular method for respiratory motion management of liver SBRT with free-breathing.

After 4D CT scanning, using combination of the targets in each respiratory phases (total ten phases) to generate internal target volume (ITV) [7], using the maximum intensity projections (MIP) [8]to delineate ITV or the medium ventilation CT to delineate the target[9] are adopted in clinical practice. The $5 \mathrm{~mm}$ uniform PTV margins have achieved widespread use [4]. CBCT corrects the inter-fraction errors pretreatment for each fraction, and the $5 \mathrm{~mm}$ PTV margin is considered adequate for intra-fraction errors, which include the amplitude variations and the baseline drift of respiratory motion during treatment. At present, to analyse intra-fractional errors, post-treatment CBCT [10-12], KV fluoroscopy [5, 13], and electromagnetics [14] are used. KV fluoroscopy is a form of two-dimensional imaging, analogous to electromagnetic tracking, which usually requires implantation of mental markers for verification. Using post-treatment $\mathrm{CBCT}$ in the dose delivering duration may not be a sufficient surrogate for the correction of intra-fraction errors. The intra-fraction CBCT can obtain a 3D image of a patient while delivering the dose, which can represent the intra-fraction errors. Ruijiang Li, et.al. [15] used intra-fraction CBCT images and confirmed that there were large intra-fraction errors in the lung SBRT. However, until now, no study has reported intra-fraction errors for liver SBRT with free-breathing using intra-fraction CBCT.

The purpose of this study was to use inter- and intra-fraction CBCT and its two-dimensional projections to quantify the amplitude variation and baseline drift of respiratory motion during liver SBRT treatment and to evaluate whether the $5 \mathrm{~mm}$ PTV margin is sufficient.

\section{Materials And Method \\ 2.1 Patients}

From June 2019 to November 2019, of the 24 included patients with liver cancer, 20 were male and 4 were female, with a median age of $58 \pm 11$ years (range: 44-82), including 20 patients with primary liver cancer and 4 patients with liver metastases. All the patients signed a consent form before radiotherapy, and this study was approved by the Ethics Committee of West China School of Medicine, Sichuan University (No.20191128). All the patients were treated with SBRT using the VAMT technique (800 cGy $\times$ 5 fractions for 11 patients, $700 \mathrm{cGy} \times 5$ fractions for 5 patients, $900 \mathrm{cGy} \times 5$ fractions for 6 patients, $1000 \mathrm{cGy} \times 5$ fraction s for 5 patients). The average dose received was $3980 \mathrm{cGy}$, and the total treatment the time was $1-3$ weeks.

\subsection{Positioning, treatment planning, treatment}

The patients were immobilized with a thermoplastic mask, which was open $5 \mathrm{~cm}$ below the xiphoid process and the navel to facilitate obtaining the patient's respiratory motion signal. All patients underwent a 4D CT scan with free-breathing using sixty-four slice spiral CT simulation (Definition AS, 
Siemens, German), with a $3 \mathrm{~mm}$ thickness. All patients were scanned with MRI for CT image fusion to assist in determining GTV. GTV was delineated in each phase (total ten phases) to obtain ITV, and a uniform $5 \mathrm{~mm}$ PTV margin $[4,13]$ was expanded around the ITV. The average intensity projections (AIP) were adopted for treatment planning and image registration with CBCT images $[13,16]$ (XVI Release 5.0.2, Elekta Versa HD, Stockholm, Sweden). Full arc or partial arc VMAT plans were designed using the $6 \mathrm{MeV}(1400 \mathrm{MU} / \mathrm{min})$ or $10 \mathrm{MeV}(2400 \mathrm{MU} / \mathrm{min})$ photon FFF mode.

\subsection{Analysis of target (i.e., the liver) position errors}

The inter-fraction CBCT was acquired when the patient setup was finished; then, the manual registration between the CBCT and planning CT were performed according to the diaphragm position [17] and the liver contour [10] (See Fig. 1). Liver position errors were obtained in the three translational directions (the $A P, M L$ and SI directions), and the rotational errors were ignored since the $6 \mathrm{D}$ treatment couch was not used. Moving the couch to correct the inter-fraction errors was done if the error was greater than or equal to $2 \mathrm{~mm}$ in any direction. СBCT scanning was performed and registered again to verify the liver position. If necessary (an error of more than $2 \mathrm{~mm}$ in any direction), the couch was moved again to correct for errors. After liver position verification, dose delivery was executed; at the same time, the intra-fraction CBCT scan was obtained for offline analysis (Scanning parameters: 120 kV, 40 mA, 40 mS, 5.5 frames/second; scanning start angle $181^{\circ}-180^{\circ}$ or $180^{\circ}-181^{\circ}$ ).

\subsection{Inter-fractions and intra-fraction liver baseline drift}

To analyse the baseline drift of the liver position, all the inter- and intra-fraction CBCT images were registered with planning $\mathrm{CT}$ offline. The vertebral bone was automatically registered to obtain the coarse results first; then, the manual fine adjustment followed (See Fig. 1). The results of the vertebral body registration represented the setup errors of the patients [11]. The correlation between the setup error and the liver position errors was analysed. The differences between the setup errors and the liver position errors were defined as the liver baseline drift. The inter-fraction and intra-fraction liver baseline drifts were defined as:

Liver baseline drift $_{\text {inter }}=$ Liver position $_{\text {inter }}-$ Setup $_{\text {inter }}$

Liver baseline drift $_{\text {intra }}=\left(\right.$ Liver position $_{\text {intra }}-$ Setup $\left._{\text {intra }}\right)-$ Liver position $_{\text {inter }}$

\subsection{Amplitude variation of respiratory motion and relative diaphragm position changes}

To quantify the amplitude variation of respiratory motion, the amplitude of respiratory motion between the 4D CT simulation and treatment fractions was compared. As for 4D CT, the amplitude of respiratory 
motion was measured between the end of expiration and end of inhalation using the MIM Maestro ${ }^{\circledR}$ system (Version 6.8.3, MIM Software Inc. Cleveland, USA). The inter- and intra-amplitude of respiratory motion were evaluated based on the two-dimensional projections of the inter- and intra-fraction CBCT in the anterior-posterior direction for a single breathing cycle.

To evaluate the stability of the liver (diaphragm) position, the relative diaphragm position was assessed at the end of expiration [10], and the pre-defined vertebrae (such as T12) in the SI direction were obtained in $4 \mathrm{D} \mathrm{CT}$ and the inter- and intra-fraction CBCT. The relative diaphragm position in the inter- and intrafraction CBCT was measured at the end of expiration based on the two-dimensional projections (fluoroscopy) in the anterior-posterior direction for a single breathing cycle. The correlation between the relative diaphragm position in a single breathing cycle and the corresponding liver position in the interand intra-fraction CBCT was analysed, which provided the evidence as to whether the relative diaphragm position was stable during the multi-breathing cycle (during the СBCT acquiring or dose delivering process).

To ensure the consistency of all the 3D image registrations and measurements in the two-dimensional projections, all the image registration and measurements were performed by two therapists with 10 years of experience and were reviewed by a physician with 20 years of experience in radiotherapy.

\subsection{Statistical method}

All data were processed and analysed using SPSS 20 and EXCEL, and correlations were analysed using Pearson correlation analysis. The PTV margin was calculated according to the formula PTV margin $=2.5$ $\Sigma+0.7 \sigma[18]$, where $\Sigma$ is the systematic error, which is the standard deviation of each patient's mean; $\sigma$ is the random error, which is the root mean square of each patient's standard deviation.

\section{Result}

A total of 103 paired inter- and intra-fraction CBCTs were obtained for 24 patients. The liver position errors, setup errors and baseline drifts of the inter-fraction are shown in Table 1. The systematic and random errors of liver position in the ML, AP, and SI were 1.71 and $1.85 \mathrm{~mm}, 8.93$ and $5.72 \mathrm{~mm}$, and 1.29 and $1.86 \mathrm{~mm}$, respectively. The corresponding PTV margin of the SI direction was $26.31 \mathrm{~mm}$. The maximum setup errors and liver position baseline drifts were in the SI direction, and the corresponding PTV margins were $24.37 \mathrm{~mm}$ and $18.50 \mathrm{~mm}$. The inter-fraction liver position errors were obviously related to the setup errors. The correlation coefficients were $0.80,0.70$, and 0.625 in the ML, SI and AP directions, respectively (details see Table 2 ). 
Table 1

Inter-fraction errors and the corresponding PTV margins (mm)

\begin{tabular}{|llllllllll|}
\hline \multirow{2}{*}{ Inter-fraction } & \multicolumn{2}{l}{ Liver position errors } & \multicolumn{3}{c}{ Setup errors } & \multicolumn{4}{c|}{ Liver position baseline drifts } \\
\cline { 2 - 11 } & ML & SI & AP & ML & SI & AP & ML & SI & AP \\
\hline$\Sigma$ & 1.71 & 8.93 & 1.29 & 2.39 & 8.28 & 1.89 & 1.25 & 6.12 & 1.41 \\
$\sigma$ & 1.85 & 5.72 & 1.86 & 2.21 & 5.24 & 1.92 & 1.64 & 4.57 & 1.79 \\
PTV & 5.57 & 26.31 & 4.54 & 7.53 & 24.37 & 6.07 & 4.27 & 18.50 & 4.79 \\
\hline
\end{tabular}

Table 2

Correlation between liver position errors and setup errors

\begin{tabular}{|c|c|c|c|c|c|c|c|}
\hline \multirow[t]{2}{*}{ Setup errors } & & \multicolumn{3}{|c|}{$\begin{array}{l}\text { Inter-fraction liver } \\
\text { position errors }\end{array}$} & \multicolumn{3}{|c|}{$\begin{array}{l}\text { Intra-fraction liver } \\
\text { position errors }\end{array}$} \\
\hline & & ML & SI & $\mathrm{AP}$ & $\mathrm{ML}$ & SI & AP \\
\hline Inter-fraction & $\mathrm{ML}$ & $0.80 *$ & -0.13 & -0.13 & & & \\
\hline \multirow[t]{2}{*}{ Setup errors } & $\mathrm{SI}$ & -0.08 & $0.70 *$ & -0.33 & & & \\
\hline & AP & 0.12 & -0.14 & $0.625^{\star}$ & & & \\
\hline Intra-fraction & $\mathrm{ML}$ & & & & $0.46 *$ & 0.22 & 0.07 \\
\hline \multirow[t]{2}{*}{ Setup errors } & $\mathrm{SI}$ & & & & 0.05 & $0.25^{\star}$ & -0.10 \\
\hline & AP & & & & 0.10 & -0.26 & 0.10 \\
\hline
\end{tabular}

After CBCT correction, the intra-fraction liver position errors were significantly reduced. The systematic and random errors in the $\mathrm{ML}, \mathrm{AP}$, and SI directions were 1.12 and $1.35 \mathrm{~mm}, 1.81$ and $2.91 \mathrm{~mm}$, and 0.94 and $1.32 \mathrm{~mm}$, respectively, with corresponding PTV margin of $3.75 \mathrm{~mm}, 6.56 \mathrm{~mm}$, and $3.28 \mathrm{~mm}$ (Table 3). Because the couch correction is based on the result of the liver position errors, there were still large interfraction setup errors. The maximum error was in the SI direction, with a systematic error of $5.87 \mathrm{~mm}$ and random error of $3.83 \mathrm{~mm}$. However, the correlation between the intra-fraction liver position errors and intra-fraction setup errors was low. Only in the ML and SI directions was there a statistical correlation; the correlation coefficients were 0.46 and 0.25 (for details, see Table 2). The systematic error and random error of the intra-fraction liver position baseline drift in the SI direction were 2.03 and $2.46 \mathrm{~mm}$, respectively, and the corresponding PTV margin was $6.8 \mathrm{~mm}$ (see Table 3). 
Table 3

Intra-fraction errors and the corresponding PTV margins (mm)

\begin{tabular}{|llllllllll|}
\hline \multirow{2}{*}{$\begin{array}{l}\text { Intra-fraction } \\
\text { errors }\end{array}$} & \multicolumn{3}{l}{ Liver position errors } & \multicolumn{2}{c}{ Setup errors } & \multicolumn{4}{c|}{ Liver position baseline drift } \\
\cline { 2 - 11 } & ML & SI & AP & ML & SI & AP & ML & SI & AP \\
\hline$\Sigma$ & 1.12 & 1.81 & 0.94 & 1.21 & 5.87 & 1.28 & 0.99 & 2.03 & 1.02 \\
$\sigma$ & 1.35 & 2.91 & 1.32 & 1.68 & 3.83 & 1.58 & 1.60 & 2.46 & 2.07 \\
PTV & 3.75 & 6.56 & 3.28 & 4.20 & 17.35 & 4.31 & 3.61 & 6.80 & 4.00 \\
\hline
\end{tabular}

The average respiratory motion in $4 \mathrm{D} \mathrm{CT}$, the inter-fraction CBCT and the intra-fraction CBCT were $13.39 \mathrm{~mm}, 13.12 \mathrm{~mm}$ and $12.09 \mathrm{~mm}$, respectively. However, the amplitude variation of respiratory motion in the same patient was considerable. Taking the amplitude of the respiratory motion in 4D CT as a reference, the amplitude variation ranged from-169.33-65.47\% in the inter-fraction CBCT and from $171.04-60.00 \%$ in the intra-fraction СВCT (see Fig. 1). The difference of the respiratory motion amplitude in 4D CT and in the inter-fraction CBCT (103 fractions) was $0.28 \pm 5.41 \mathrm{~mm} ; 66$ of 103 fractions $(64 \%)$ were within $5 \mathrm{~mm}$. The difference of the respiratory motion amplitude in 4D CT and in the intra-fraction CBCT (103 fractions) was $1.03 \pm 4.35 \mathrm{~mm}$, with $69 \%$ of fractions being within $5 \mathrm{~mm}$ (See Fig. 2). The relative diaphragm position (measured by the two-dimensional projections at the anterior-posterior direction for a single breathing cycle) was not statistically related to the baseline drift in the inter-fraction nor the intra-fraction (See Table 4). The respiratory motion amplitude had a weak statistical correlation to the liver position error in the SI direction both in the inter-fraction and the intra-fraction (See supplementary file 2).

Table 4

Correlation between liver position drifts and the diaphragm relative position changes in $2 \mathrm{D}$ projections

\begin{tabular}{|c|c|c|c|c|c|c|c|}
\hline & & \multicolumn{3}{|c|}{$\begin{array}{l}\text { Inter-fraction liver } \\
\text { position drift }\end{array}$} & \multicolumn{3}{|c|}{$\begin{array}{l}\text { Intra-fraction liver } \\
\text { position drift }\end{array}$} \\
\hline & & ML & SI & AP & $\mathrm{ML}$ & SI & $\mathrm{AP}$ \\
\hline \multirow{4}{*}{$\begin{array}{l}\text { The diaphragm } \\
\text { relative position }\end{array}$} & Coefficient & -0.07 & 0.08 & -0.07 & & & \\
\hline & $P_{\text {value }}$ & 0.49 & 0.46 & 0.47 & & & \\
\hline & Coefficient & & & & -0.01 & -0.03 & 0.18 \\
\hline & $P_{\text {value }}$ & & & & 0.96 & 0.79 & 0.06 \\
\hline
\end{tabular}

\section{Discussion}


Chan $\mathrm{M}$ reported [9] that there was no significant difference between the use of $3 \mathrm{D}$ CBCT and $4 \mathrm{D}$ CBCT in liver radiotherapy. To reduce the $\mathrm{CBCT}$ scanning time, we used $3 \mathrm{D} C \mathrm{CBCT}$ image guidance. Markus Oechsner reported [16]that AIP is more suitable for 3D CBCT than MIP. Bedos $L$ et al. also believed that [13] CBCT was an average image and that registration with the planned AIP image can represent the patient's breathing pattern, so we used AIP as the reference image for registration with 3D CBCT images for liver SBRT. In the inter- and intra-fraction CBCT, the liver contour can be clearly displayed (Fig. 1), and the registration error of the two therapists (inter-observer error) was less than $1 \mathrm{~mm}$ in the $\mathrm{ML}, \mathrm{SI}$ and $\mathrm{AP}$ directions (SD).

Our results showed that the liver position and the setup errors for the inter-fraction were considerable, and there was a significant correlation between the setup errors and the liver position errors. Meanwhile, the liver baseline drifts demonstrated large systematic and random errors in the ML, SI and AP directions, which were $1.25 \mathrm{~mm}$ and $1.64 \mathrm{~mm}, 6.12 \mathrm{~mm}$ and $4.57 \mathrm{~mm}$, and $1.41 \mathrm{~mm}$ and $1.79 \mathrm{~mm}$, respectively. Case RB et al. [10] found that in patients with free breathing, the systematic error and random error of the liver position baseline drifts in the $\mathrm{ML}, \mathrm{SI}$ and $\mathrm{AP}$ directions were $1.5 \mathrm{~mm} / 1.8 \mathrm{~mm}, 3.1 / 3.6 \mathrm{~mm}$, and $1.6 / 2.7 \mathrm{~mm}$, respectively. The error in the SI direction in that report was significantly smaller than ours. The main reason for this difference was that in that report, the patients planning used end-expiratory CT and the same respiratory phase $\mathrm{CBCT}$ image for registration. In our study, the free breathing average CBCT and AIP CT were used for registration. Dhont $J$ reported [19] that the liver baseline drifts between inter-fractions were $1.6 \pm 1.3,3.0 \pm 1.2 \mathrm{~mm}$, and $2.1 \pm 1.4 \mathrm{~mm}$ in the $\mathrm{ML}, \mathrm{SI}$, and AP directions, respectively.

In our study, the liver position errors and baseline drifts in the intra-fraction were significantly reduced compared to those in the inter-fraction, indicating that using CBCT to correct the liver position was effective. The baseline drifts in the $\mathrm{ML}, \mathrm{SI}$, and AP directions were $0.99 / 1.60 \mathrm{~mm}, 2.03 / 2.46 \mathrm{~mm}$, 1.02/2.07 mm, respectively, similar to the Case RB report using CВCT (1.2/2.2 mm 1.4/3.0 mm 1.0/1.9 mm, respectively) [10]. Bedos L. et. al. [13]used a KV plain film to acquire the end-expiratory image to analyse the patient's intra-fraction errors and found that the $99 \%$ intra-fraction error in the $\mathrm{SI}$ direction was within $5 \mathrm{~mm}$, which was smaller than in our study. However, the images were collected before the treatment beam was on, and the acquisition time was limited, which cannot represent intrafraction errors. Another report showed that the medians (ranges) of the baseline drifts were 1.87 (0.0612.04) $\mathrm{mm}, 0.35(0-3.39) \mathrm{mm}$ and $1(0.02-7.21) \mathrm{mm}$ in the SI, LR and AP directions, respectively [20]. However, this study defined the baseline as the average position in the breathing cycle during treatment. In our study, the baseline was defined in $4 \mathrm{D} \mathrm{CT}$, and the intra-fraction CBCT was used to evaluate intrafraction errors. The average frame number of the intra-fraction CBCT was 467 frames, and the average acquisition time was 84.9 seconds, which can include approximately 20 breathing cycles. Xu Q et al. [21]used liver markers to evaluate the baseline displacement, and they found that the baseline displacement of ML, AP and SI were $2.1 \pm 2.3 \mathrm{~mm}, 2.9 \pm 2.8 \mathrm{~mm}$, and $6.4 \pm 5.5 \mathrm{~mm}$, respectively. By using $4 \mathrm{D} \mathrm{CT}$ as a reference, the baseline drifts were significant during treatment according to these reports and our findings. In other words, the vertebral bone cannot be used for position verification for liver SBRT due to the considerable liver baseline drifts. 
CBCT using two-dimensional projections (fluoroscopy) can evaluate the stability of respiratory motion during the entire respiratory cycle. Dhont $\mathrm{J}$ et al. [19] used a single metal marker to analyse the SI direction of respiratory motion The amplitude change over $5 \mathrm{~mm}$ was $53 \%$ for the inter-fraction and $28 \%$ for the intra-fraction. Shimohigashi Y S et al. [22] reported that in using abdominal pressure, the average movement in the SI direction was $5.3 \mathrm{~mm}$, and the maximum value was $14.8 \mathrm{~mm}$. The medians (ranges) of the intra-fraction amplitude variation across all patients were $4.3(1.6-6.0) \mathrm{mm}, 0.5(0.2-2.2) \mathrm{mm}$ and $1.5(0.3-3.3) \mathrm{mm}$ in the SI, LR and AP directions, respectively [20]. In the current study, the variation of the respiratory motion amplitude among $4 \mathrm{D} \mathrm{CT}$, the inter-fraction CBCT and the intra-fraction CBCT in the SI direction was small (the average amplitude was $13.39 \mathrm{~mm}, 13.12 \mathrm{~mm}$ and $12.09 \mathrm{~mm}$, respectively), indicating that the overall patient respiratory motion amplitude was relatively stable. However, for individuals, there was a large difference. The variation of the respiratory motion amplitude for the intrafraction CBCT ranged from $-171.04-60.00 \%$, and the overall difference was $1.03 \pm 4.35 \mathrm{~mm}$. In $69 \%$ of cases, the overall difference was less than $5 \mathrm{~mm}$. However, because we only evaluated a single breathing cycle using fluoroscopy, this cannot represent the real respiratory motion state during the whole treatment process (it was a snapshot image like 4D CT). The respiratory motion amplitude and baseline may change during the treatment process, and this uncertainty was more obvious when the movement was greater than $7 \mathrm{~mm}$ [19]. Meanwhile, the report noted that the accuracy of the 4D CT phase classification according to amplitude was higher than that of the phase classification according to time [23]. Therefore, if $4 \mathrm{D} \mathrm{CT}$ was used, the change in the motion amplitude and the baseline drifts should be completely evaluated and understood when determining the PTV margin.

Considering the baseline drifts, Dhont $\mathrm{J}$ et al. recommended the $8 \mathrm{~mm}$ PTV margin for liver [19]. Worm ES et al. [24]reported that when the target area was delineated using 4D CT with moderate ventilation, a $10 \mathrm{~mm}$ PTV margin in the SI direction and $5 \mathrm{~mm}$ AP and ML directions should be used. Case RB [10] found that the patient's respiratory motion changed little during radiotherapy, and the results were not statistically related to the time of radiotherapy. Although there were large variations of the respiratory motion amplitude in our study, this cannot be a main reason to expand the PTV margin. Fortunately, the respiratory movement is a 3D movement around the baseline mainly in the SI direction. The baseline drift was a systematic error, which can induce a systematic dose deviation, and the amplitude changes were random errors, which can blur the dose around the target edge. Therefore, the baseline drift in the intrafraction is the most important error when determining the PTV margin. According to the results in our study, the $5 \mathrm{~mm}$ PTV margin was sufficient in the ML and AP directions, but there needs to be a $6.80 \mathrm{~mm}$ PTV margin in the SI direction.

Since metal markers were not implanted in or near the tumour, the three-dimensional position deviation of the tumour cannot be analysed using two-dimensional projections. Therefore, implanted metal markers [25]or MR [3]should be used to obtain more soft tissue information and to further analyse the respiratory motion amplitude changes and baseline drifts. If we want to comprehensively analyse the variation of respiratory motion, we need automatic methods to evaluate the two-dimensional projections during whole treatments. 


\section{Conclusions}

When using intra-fraction $\mathrm{CBCT}$, significant amplitude variations and baseline drifts of respiratory motion were found compared with those in $4 \mathrm{D} \mathrm{CT}$. The relative diaphragm position in a single breathing cycle cannot reflect the liver position during dose delivery. At least a $6.8 \mathrm{~mm}$ PTV margin in the SI direction is recommended to compensate for the baseline drift. The dose effect caused by the respiratory motion amplitude changes and baseline drifts during dose delivery should be studied further.

\section{List Of Abbreviations}

CBCT Cone beam computed tomography

SBRT Stereotactic Body Radiation Therapy

4D CT Four-dimensional computed tomography

PTV Planning target volume

KV Kilovoltage

MR Magnetic resonance

EPID Electron portal imaging device

DIBH Deep inspiration breath-hold

ITV Internal target volume

MIP Maximum intensity projections

GTV Gross target volume

XVI X-Ray Volume Imaging

VMAT Volumetric modulated arc therapy

AIP Averaged intensity projections

MRI Magnetic resonance imaging

\section{Declarations}

\section{Ethics approval and consent to participate}


All the patients signed a consent form before radiotherapy, and this study was approved by the Ethics Committee of West China School of Medicine, Sichuan University (No.20191128).

\section{Consent for publication}

All authors reviewed the manuscript and consent for publication.

\section{Availability of data and materials}

The data and materials of this manuscript is available if necessary.

\section{Competing interests}

The authors declare that they have no competing interests.

\section{Funding}

This work was supported by the 1.3.5 Project for Disciplines of Excellence, West China Hospital, Sichuan University (ZYJC18008).

\section{Authors' contributions}

Lu Zeng collected data and drafted the manuscript. Xin Wang designed the patients' treatment planning and reviewed the image registration. Jidan Zhou, Pan Gong, Xuetao Wang, Xiaohong Wu, Zhonghua Deng, and Bin Li helped to collect the data. Renming Zhong designed the study, revised and final approved the manuscript. All authors read and confirmed the manuscript.

\section{Acknowledgements}

Not applicable

\section{References}

1. Yang $M$ and R Timmerman. Stereotactic Ablative Radiotherapy Uncertainties: Delineation, Setup and Motion. Semin Radiat Oncol 2018; 28:207-217.

2. Li W Z, Z W Liang, Y Cao, et al. Estimating intrafraction tumor motion during fiducial-based liver stereotactic radiotherapy via an iterative closest point (ICP) algorithm. Radiat Oncol 2019; 14:185. 
3. Rosenberg S A, L E Henke, N Shaverdian, et al. A Multi-Institutional Experience of MR-Guided Liver Stereotactic Body Radiation Therapy. Adv Radiat Oncol 2019; 4:142-149.

4. Gargett $M, C$ Haddad, $A$ Kneebone, et al. Clinical impact of removing respiratory motion during liver SABR. Radiat Oncol 2019; 14:93.

5. Poulsen P R, G Murtaza, E S Worm, et al. Simulated multileaf collimator tracking for stereotactic liver radiotherapy guided by kilovoltage intrafraction monitoring: Dosimetric gain and target overdose trends. Radiother Oncol 2020; 144:93-100.

6. Zhong R, J Wang, X Jiang, et al. Hypofraction radiotherapy of liver tumor using cone beam computed tomography guidance combined with active breath control by long breath-holding. Radiother Oncol 2012; 104:379-85.

7. Borm K J, M Oechsner, M Wiegandt, et al. Moving targets in 4D-CTs versus MIP and AIP: comparison of patients data to phantom data. BMC Cancer 2018; 18:760.

8. Bradley J D, A N Nofal, I M El Naqa, et al. Comparison of helical, maximum intensity projection (MIP), and averaged intensity (AI) 4D CT imaging for stereotactic body radiation therapy (SBRT) planning in lung cancer. Radiother Oncol 2006; 81:264-8.

9. Chan M, C L Chiang, V Lee, et al. Target localization of 3D versus 4D cone beam computed tomography in lipiodol-guided stereotactic radiotherapy of hepatocellular carcinomas. PLoS One 2017; 12:e0174929.

10. Case R B, J J Sonke, D J Moseley, et al. Inter-and intrafraction variability in liver position in nonbreath-hold stereotactic body radiotherapy. Int J Radiat Oncol Biol Phys 2009; 75:302-8.

11. Zhang $T, W$ Wang, $Y$ Li, et al. Inter- and intrafractional setup errors and baseline shifts of fiducial markers in patients with liver tumors receiving free-breathing postoperative radiation analyzed by cone-beam computed tomography. J Appl Clin Med Phys 2014; 15:4914.

12. Case R B, D J Moseley, J J Sonke, et al. Interfraction and intrafraction changes in amplitude of breathing motion in stereotactic liver radiotherapy. Int J Radiat Oncol Biol Phys 2010; 77:918-25.

13. Bedos L, O Riou, N Aillères, et al. Evaluation of reproducibility of tumor repositioning during multiple breathing cycles for liver stereotactic body radiotherapy treatment. Rep Pract Oncol Radiother 2017; 22:132-140.

14. Nankali S, E S Worm, R Hansen, et al. Geometric and dosimetric comparison of four intrafraction motion adaptation strategies for stereotactic liver radiotherapy. Phys Med Biol 2018; 63:145010.

15. Li R, B Han, B Meng, et al. Clinical implementation of intrafraction cone beam computed tomography imaging during lung tumor stereotactic ablative radiation therapy. Int J Radiat Oncol Biol Phys 2013; 87:917-23.

16. Oechsner M, B Chizzali, M Devecka, et al. Registration uncertainties between 3D cone beam computed tomography and different reference $\mathrm{CT}$ datasets in lung stereotactic body radiation therapy. Radiat Oncol 2016; 11:142.

17. Yang J, J Cai, $\mathrm{H}$ Wang, et al. Is diaphragm motion a good surrogate for liver tumor motion? Int J Radiat Oncol Biol Phys 2014; 90:952-8. 
18. van Herk M. Errors and margins in radiotherapy. Semin Radiat Oncol 2004; 14:52-64.

19. Dhont J, J Vandemeulebroucke, M Burghelea, et al. The long- and short-term variability of breathing induced tumor motion in lung and liver over the course of a radiotherapy treatment. Radiother Oncol 2018; 126:339-346.

20. Liang Z, H Liu, J Xue, et al. Evaluation of the intra- and interfractional tumor motion and variability by fiducial-based real-time tracking in liver stereotactic body radiation therapy. J Appl Clin Med Phys 2018; 19:94-100.

21. Xu Q, G Hanna, J Grimm, et al. Quantifying rigid and nonrigid motion of liver tumors during stereotactic body radiation therapy. Int J Radiat Oncol Biol Phys 2014; 90:94-101.

22. Shimohigashi $Y, R$ Toya, T Saito, et al. Tumor motion changes in stereotactic body radiotherapy for liver tumors: an evaluation based on four-dimensional cone-beam computed tomography and fiducial markers. Radiation Oncology 2017; 12.

23. Thengumpallil S, J F Germond, J Bourhis, et al. Impact of respiratory-correlated CT sorting algorithms on the choice of margin definition for free-breathing lung radiotherapy treatments. Radiother Oncol 2016; 119:438-43.

24. Worm E S, M Høyer, W Fledelius, et al. Three-dimensional, time-resolved, intrafraction motion monitoring throughout stereotactic liver radiation therapy on a conventional linear accelerator. Int $\mathrm{J}$ Radiat Oncol Biol Phys 2013; 86:190-7.

25. Bertholet J, E S Worm, W Fledelius, et al. Time-Resolved Intrafraction Target Translations and Rotations During Stereotactic Liver Radiation Therapy: Implications for Marker-based Localization Accuracy. Int J Radiat Oncol Biol Phys 2016; 95:802-9.

\section{Figures}


Figure 1. Liver position error registration and setup error registration

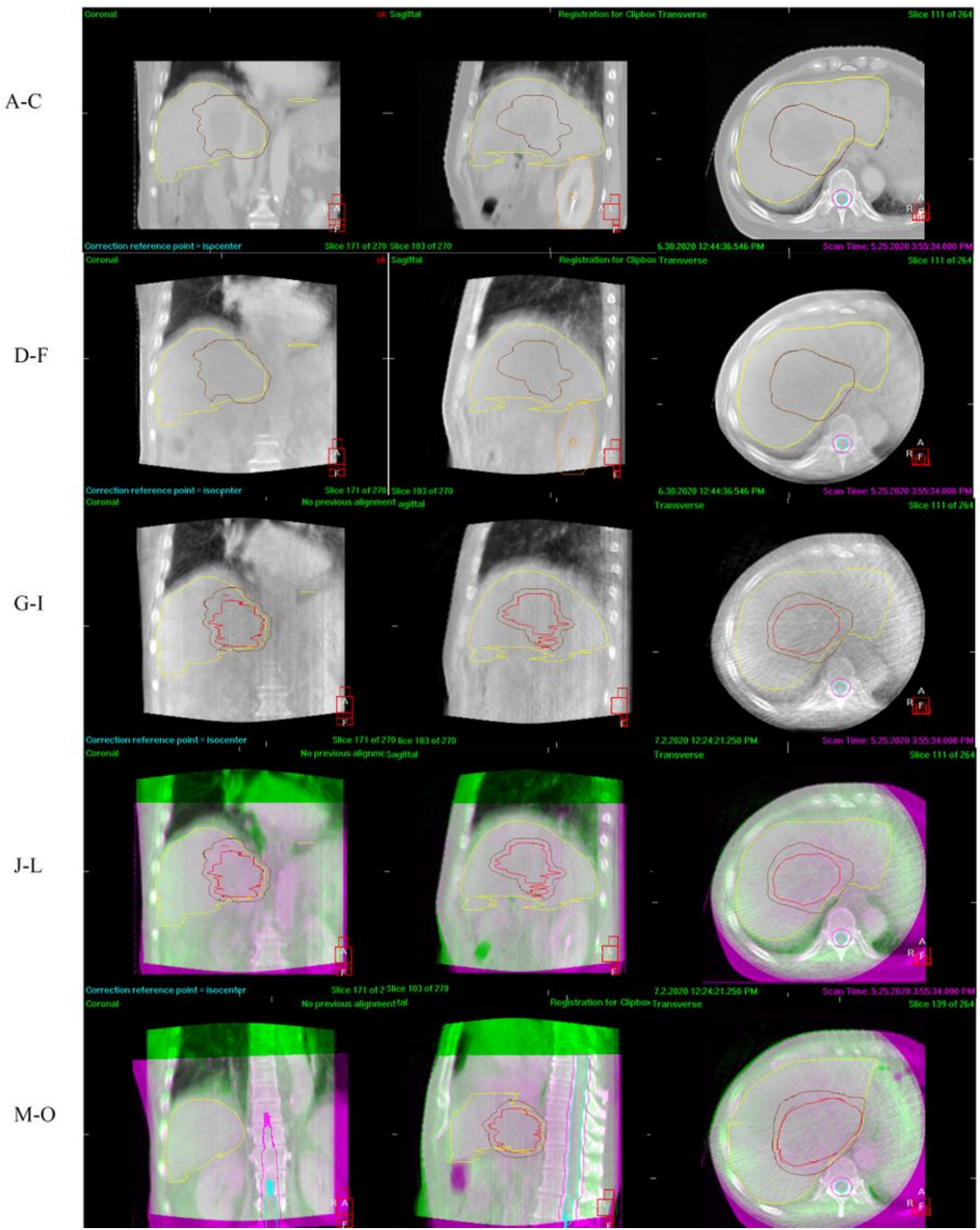

\section{Figure 1}

Liver position error registration and setup error registration. A-C refer to the planning CT; D-F refer to the inter-fraction $\mathrm{CBCT}$; G-I refer to the intra-fraction $\mathrm{CBCT}$; J-L refer to the liver position error registration and $\mathrm{M}-\mathrm{O}$ refer to setup error registration in the intra-fraction $\mathrm{CBCT}$. 


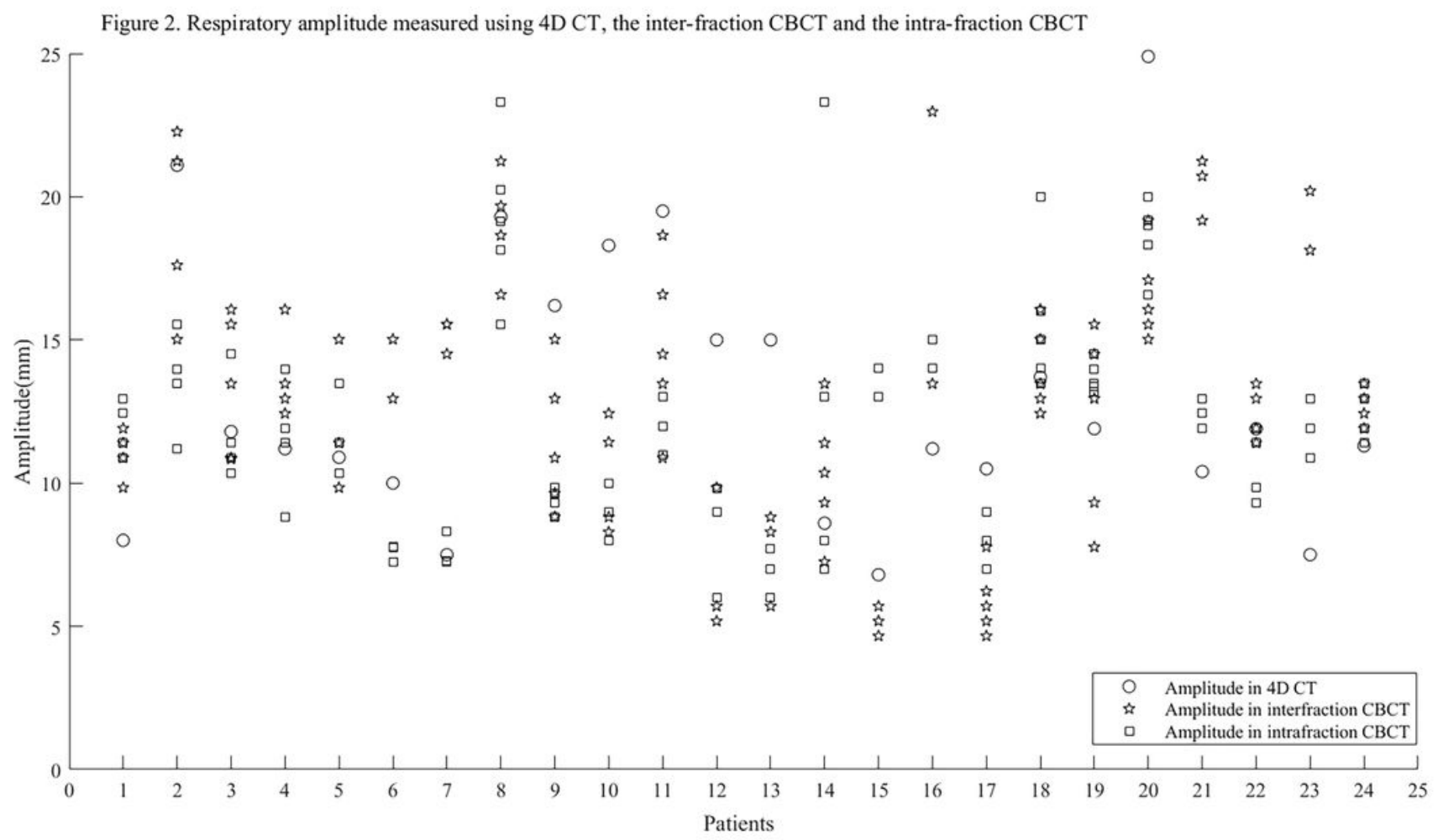

Figure 2

Respiratory amplitude measured using 4D CT, the inter-fraction CBCT and the intra-fraction CBCT.

\section{Supplementary Files}

This is a list of supplementary files associated with this preprint. Click to download.

- supplementaryfile1.docx 